\title{
Modeling and Simulation of Power Yield in Chemical and Electrochemical Systems
}

\author{
S. Sieniutycz and P.Kuran \\ Faculty of Chemical and Process Engineering, Warsaw University of Technology, \\ 1 Waryńskiego Street, 00-645 Warsaw (Poland) \\ Phone 00-48-22-256340; fax: 00-48-22-251440, e-mail: sieniutycz@ichip.pw.edu.pl
}

\begin{abstract}
Fuel cells are treated as flow engines driven by fluxes of chemical reagents and electrochemical mechanism of electric current generation. Analyzed are performance curves of a SOFC system, power limits and the effect of typical design and operating parameters on the cell performance. The theory combines a recent formalism worked out for chemical machines with the Faraday's law which determines the intensity of the electric current generation. Steady-state model of a hightemperature SOFC is considered, which refers to constant chemical potentials of incoming hydrogen fuel and oxidant. Lowering of the cell voltage below its reversible value is attributed to polarizations and imperfect conversions of reactions. A power formula summarizes effect of transport laws, irreversible polarizations and efficiency of power yield. Reversible electrochemical theory is extended to the case with dissipative chemical reactions; this case includes systems with incomplete conversions, characterized by "reduced affinities" and an idle run voltage. Effect of incomplete conversions is modeled by assuming that substrates can be remained after the reaction and that side reactions may occur. Optimum and feasibility conditions are discussed for some important process parameters such as the efficiency, power output, and electric current density. Calculations of maximum power show that the data differ for power generated and consumed. These data provide bounds for SOFC energy generators, which are more exact and informative than classical reversible bounds for electrochemical transformation.
\end{abstract}

\section{Key words}

Power limits, entropy, chemical engines, fuel cells.

\section{Introduction}

In a previous work [1] we modelled power production and its limits in purely thermal systems with finite rates. In particular, radiation engines were analyzed as nonlinear systems governed by laws of thermodynamics and transport. Temperatures $T$ of resource media were only necessary variables to describe these systems. However, chemical engines and fuel cells are more general systems in which both temperatures $T$ and chemical potentials $\mu_{\mathrm{k}}$ are essential. A chemical engine is schematized in Fig. 1, whereas a solid oxide fuel cell SOFC in Fig.2. Fuel cells (FC) are electrochemical flow engines. Their role for environmental protection cannot be underestimated. The main advantage of fuel cells in comparison to heat engines is that their efficiency is not a major function of device size.

A fuel cell continuously transforms a part of chemical energy into electrical energy by consuming fuel and oxidant. Fuel cells are electrochemical flow engines propelled by fluxes of both energy and substances.

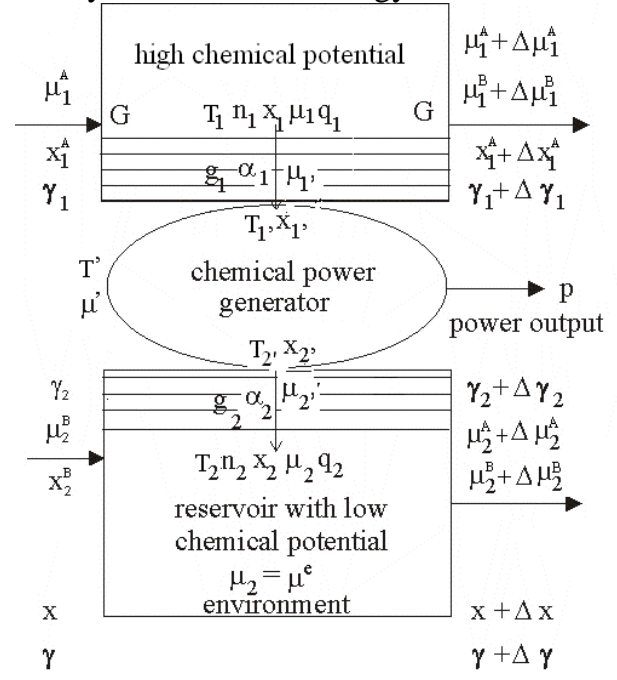

Fig.1. A scheme of chemical flow engine.

The symbols in Fig. 1 describe: $\mu$ - chemical potential of active component of fuel $\left[\mathrm{Jmol}^{-1}\right], \quad \gamma$-cumulative conductance of the system $\left[\mathrm{Js}^{-1} \mathrm{~K}^{-\mathrm{a}}\right], x$ - molar fraction of active component in the fuel, $T_{1}$ and $T_{2}$ - bulk temperatures of reservoirs $[\mathrm{K}], T_{1}$, and $T_{2}$, - temperatures of fluid circulating in the engine $[\mathrm{K}], T^{e}$ - constant temperature of environment $[\mathrm{K}], T^{\prime}$ - Carnot temperature $[\mathrm{K}], \alpha$ - heat coefficient $\left[\mathrm{Jm}^{-2} \mathrm{~s}^{-1} \mathrm{~K}^{-1}\right], q$ - heat flux $\left[\mathrm{Js}^{-1}\right]$, $g_{1}$ and $g$ - partial and overall conductances $\left[\mathrm{mols}^{-1}\right]$ ).

Basic structure of fuel cells includes electrolyte layer in contact with a porous anode and cathode on either side. Gaseous fuels are fed continuously to the anode (negative electrode) compartment and an oxidant (i.e., oxygen from air) is fed continuously to the cathode (positive electrode) compartment. Electrochemical reactions take place at the electrodes to produce an electric current. Basic reaction is 
the electrochemical oxidation of fuel, usually hydrogen, and the reduction of the oxidant, usually oxygen. This principle makes a fuel cell similar to a chemical engine. In a FC process in Fig. 2 streams of fuel $\left(\mathrm{H}_{2}\right)$ and oxidant $\left(\mathrm{O}_{2}\right)$ interact; the process is propelled by diffusive and/or convective fluxes of heat and mass, transferred through the cell 'conductances' or boundary layers. The energy flux (power) is created in the cell generator which exploits the fuel stream contacting with the anode and the oxidant stream contacting with the catode. Both electrodes are separated by the electrolyte. As in typical thermal machines and radiation engines [2]-[7] both transfer mechanisms and properties of conducting layers influence a rate of power yield.

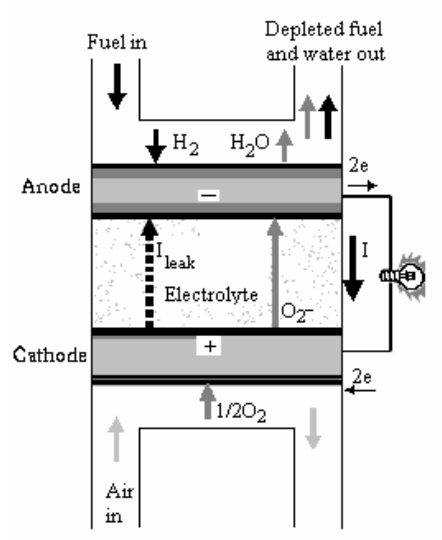

Fig. 2. Principle of a solid oxide fuel cell

Fuel cell systems working in the power yield mode are electrochemical flow engines propelled by chemical reactions. Their performance is determined by magnitudes and directions of participating streams and by mechanism of electric current generation. Voltage lowering in a cell below its reversible value is a good measure of the cell imperfection.

The goals of the present paper include: (a) formulation of a thermo-electro-chemical model for imperfect fuel cells, especially for those with incomplete chemical conversions, (b) implementation of the model to simulate the behaviour of high- $T$ solid oxide fuel cells, (c) prediction of various losses of the voltage and their effect on the cell performance, and (d) application of fuel cell characteristics for the power limits quantification.

\section{Thermodynamics of Power Generation}

Knowledge of operational voltage is required to define a cell efficiency as the ratio $\chi=V / E$, where $E$ is the reversible cell voltage or the equilibrium cell potential. For the power density in terms of $\chi$ one has $p=i E \chi$ or $p$ $=\chi^{\text {prev }}$, which means that this efficiency is equal to the ratio of the actual power to the maximum reversible power. This definition links the fuel cell efficiency with the second law, and stresses substantial role of the operational voltage. Assume that all incoming streams (those with "higher" Gibbs flux $G_{\text {in }}=G_{1}$ ') represent a common phase of "substrates" (all system's components in the state before the chemical transformation, index 1'). All outgoing streams (those with "lower" Gibbs flux $G_{\text {out }}$ $=G_{2}$, ) represent the common phase of "products" (all system components in the state after the transformation, index 2'). The power expression follows from entropy conservation and energy balance in the reversible part of the system. For an isothermal reactor power yield is

$$
\begin{aligned}
& p=\mu_{1_{1}} \dot{n}_{1^{\prime}}+\mu_{2_{1^{\prime}}} \dot{n}_{1_{1^{\prime}}}+\ldots \mu_{i_{1^{\prime}}} \dot{n}_{i_{1^{\prime}}} \ldots .+\mu_{m_{1^{\prime}}} \dot{n}_{m_{1^{\prime}}} \\
& -\mu_{2_{2^{\prime}}} \dot{n}_{1_{2^{\prime}}}-\mu_{2_{2^{\prime}}} \dot{n}_{2_{2^{\prime}}} \ldots-\mu_{i_{2^{\prime}}} \dot{n}_{i_{2^{\prime}}} \ldots .-\mu_{m_{2^{\prime}}} \dot{n}_{m_{2^{\prime}}}
\end{aligned}
$$

This formula shows that, in a steady and isothermal process, power yield of a chemical engine system is the difference between the input and output flux of the Gibb's function [8]-[11]. We can transform Eq, (1) to a pronouncing form of Eq. (2) below, specific to the case of a complete conversion. In this case the components are numbered such that species $1,2 \ldots i$ are substrates and species $i+1, i+2 \ldots \mathrm{m}$ are products. Total power yield of an isothermal multi-reaction process takes the form

$$
\begin{aligned}
& p=\sum_{j=1}^{R}\left\{p_{j}\right\}=\sum_{j=1}^{R}-\left\{\mu_{1_{1}} v_{1 j}+\mu_{2_{1}}, v_{2 j}+\ldots \mu_{i_{1}} v_{i j}\right. \\
& \left.\left.+\mu_{i+1_{2}} v_{i+1 j}+. \mu_{m-1_{2}}, v_{m-1 j} . .+\mu_{m_{2}}, v_{m j}\right)\right\} \dot{n}_{j}
\end{aligned}
$$

Quantities $\dot{n}_{j}$ are molar chemical fluxes of reagents, i.e. products of the electrode surface area $F$ and heterogeneous rates, $r_{\mathrm{j}}$. In the case of complete conversion, power yield from the unit electrode area equals the sum of products of the affinity driving forces and the reaction rates

$$
p=\sum_{j=1}^{R}\left\{\tilde{\mathrm{A}}_{j} \dot{n}_{j}\right\}=F \sum_{j=1}^{R}\left\{\tilde{\mathrm{A}}_{j} r_{j}\right\}
$$

Yet, the assumption about the complete transformation of substrates into products can be relaxed, and the present paper shows how this can be done for fuel cells. By considering the chemistry of systems with power production and transport phenomena one can quantitatively estimate effects of incomplete conversions. The related formula resembles the one which describes the effect of the internal entropy production within these systems [11]. For a single isothermal chemical reaction the corresponding power formula which generalizes Eq. (3) to include effect of incomplete conversions can be written in the form

$$
p=\left(\Pi_{1^{\prime}}-\Xi \zeta_{2^{\prime}}\right) \dot{\eta}_{1^{\prime}}=-\frac{i A}{n_{e} F} \Delta g^{e f f}(T, p)=-\Delta \dot{G}^{e f f},
$$

where primed quantities refer to the inputs and outputs of the chemically active zone and $\dot{Q}_{1^{\prime}}$ is the total heat flux (involving the sensible heat flux, $q_{1}$, and the sum of products of partial entropies and fluxes of species multiplied by the temperature $T$ ), $\Pi_{1}$, is "one-way chemical affinity" attributed to reactants with known chemical potentials $[8,11], \dot{n}_{1^{\prime}}$ is the (positive) chemical 
flux defined as the product of the heterogeneous reaction rate and the electrode area. Internal imperfection functions, $\Phi$ and $\Xi$, are respectively related to internal entropy production and incomplete conversion. The fraction $\Xi$ is the reciprocity of coefficient $\Psi$ introduced in [11]; they both characterize detrimental increase of chemical potentials of reaction products caused by their dilution by remaining reactants.

Power formula of Eq. (4) generalizes the idealized power of an "endoreversible" system (with $\Xi=1$ ) in which case difference $\Pi_{1},-\Pi_{2}$, is chemical affinity or $-\Delta g$. This is the chemical component of power, which describes power yield caused by chemical flux $n_{1}$. Electrochemical power is generated with non-ideal chemical efficiency $\xi=\Pi_{1},-\Xi \Pi_{2}$. For the simplest reaction, $1 \Leftrightarrow 2, \xi=\mu_{1}$, $\Xi \mu_{2}$, which is lower than $\mu_{1},-\mu_{2}$. Effectively, in the engine mode where $\Xi=<1$, the system with internal imperfections, behalves as it would operate with a decreased affinity of an effective value $\Pi_{1},-\Xi \Pi_{2}$. Of course, power production is decreased by this imperfection. Total entropy balance of the system leads to total entropy source $\sigma_{\mathrm{s}}$ as the difference between outlet and inlet entropy fluxes. Its qualitative characteristics and link with the power yield is shown in Fig.3.
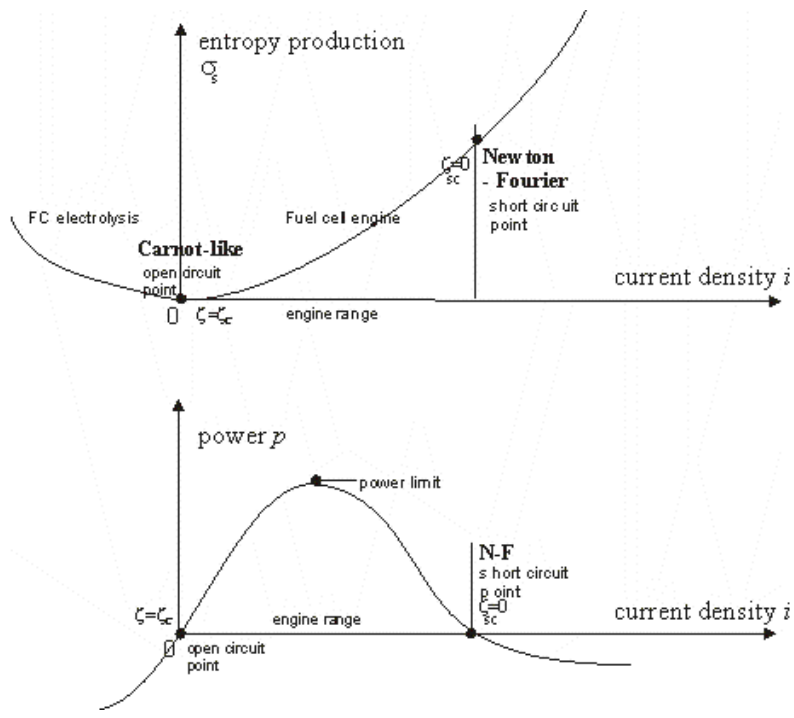

Fig. 3. Qualitative picture of entropy and power production in fuel cells in terms of the current density.

\section{Chemical Power Systems}

The thermodynamic approach can be applied to chemical and electrochemical engines. Here we shall make only a few basic remarks. In chemical engines mass transports participate in transformation of chemical affinities into mechanical power $[9,10]$. Yet, as opposed to thermal machines, in chemical ones generalized streams or reservoirs are present, capable of providing both heat and substance. Large streams or infinite reservoirs assure constancy of chemical potentials. Problems of extremum power (maximum of power produced and minimum of power consumed) are static optimization problems. For a finite "upper stream", however, amount and chemical potential of an active reactant decrease in time, and considered problems are those of dynamic optimization and variational calculus. Because of the diversity and complexity of chemical systems the area of power producing chemistries is extremely broad.

The simplest model of power producing chemical engine is that with an isothermal isomerization reaction, $A_{1}-A_{2}$ $=0[3,9]$. Power expression and efficiency formula for the chemical system follow from the entropy conservation and energy balance in the power-producing zone of the system ('active part'). In an 'endoreversible chemical engine' total entropy flux is continuous through the active zone. When a formula describing this continuity is combined with energy balance we find in an isothermal case

$$
p=\left(\mu_{1^{\prime}}-\mu_{2^{\prime}}\right) n_{1}
$$

where the feed flux $n_{1}$ equals to $n$, an invariant molar flux of reagents. Process efficiency $\zeta$ is defined as power yield per molar flux, $n$. This efficiency is identical with the chemical affinity of our reaction in the chemically active part of the system. While $\zeta$ is not dimensionless, it describes correctly the system. In terms of a Carnot variable, $\mu$, defined in [1]

$$
\zeta=\mu^{\prime}-\mu_{2}
$$

For a steady engine the following function describes chemical Carnot control $\mu$ ' in terms of fuel flux $n_{1}$ and its mole fraction $x$

$$
\mu^{\prime}=\mu_{2}+\zeta_{0}+R T \ln \left(\frac{x_{1}-n_{1} g_{1}^{-1}}{n_{1} g_{2}^{-1}+x_{2}}\right)
$$

As Eq. (6) is valid, Eq. (7) also characterizes the efficiency control in terms of $\mathrm{n}$ and fuel fraction $x$.

Equation (7) shows that an effective concentration of the reactant in upper reservoir $x_{\text {leff }}=x_{1}-g_{1}^{-1} n$ is decreased, whereas an effective concentration of the product in lower reservoir $x_{2 \mathrm{eff}}=x_{2}+g_{2}^{-1} n$ is increased due to the finite mass flux. Therefore efficiency $\zeta$ decreases nonlinearly with $n$. When effect of resistances $g_{k}^{-1}$ is ignorable or flux $n$ is very small, reversible Carnot-like chemical efficiency, $\zeta_{\mathrm{C}}$, is attained. The power function, described by the product $\zeta(n) n$, exhibits a maximum for a finite value of the fuel flux, $n$.

Application of Eq. (7) to the Lagrangian relaxation path leads to a work functional

$$
W=-\int_{\tau_{1}^{i}}^{\tau_{1}^{f}}\left\{\zeta_{0}+R T \ln \left(\frac{X /(1+X)+d X / d \tau_{1}}{x_{2}-j d X / d \tau_{1}}\right)\right\} \frac{d X}{d \tau_{1}} d \tau_{1}
$$

whose maximum describes the dynamical limit of the system. Here $X=x /(1-x)$ and $\mathrm{j}$ equals the ratio of upper to lower mass conductance, $g_{1} / g_{2}$. The path optimality condition may be expressed as the constancy of the following Hamiltonian 


$$
H(X, \dot{X})=R T \dot{X}^{2}\left(\frac{1+X}{X}+\frac{j}{x_{2}}\right)
$$

For low rates and large concentrations $X$ (mole fractions $x_{1}$ close to the unity) optimal relaxation rate of the fuel resource is approximately constant. Yet, in an arbitrary situation optimal rates are state dependent so as to preserve the constancy of $H$ in Eq. (9). Extensions of Eq. (7) are available for multicomponent and multireaction systems [11].

\section{Effect of Transports on Power Yield}

We continue our considerations in the case of a simple isomerisation reaction $A_{1}-A_{2}=0$. Transported energy and components drive power generation in fuel cells. Interestingly, there exists a formal link between the mathematics of thermal engines and fuel cells. To show this link let us note that the power producing force in an endoreversible thermal engine equals $T_{1},-T_{2}$, Whereas the propelling force in the simplest electrochemical engine is $\mu_{1},-\mu_{2}$. For bulks of the streams or reservoirs related differences of temperature and electrochemical potential are $T_{1}-T_{2}$ and $\mu_{1}-\mu_{2}$. Since the deviations of $T_{1}$, and $\mu_{1}$, from $T_{1}$ and $\mu_{1}$ are of purely dissipative origin and the bulk differences $T_{1}-T_{2}$, and $\mu_{1}-\mu_{2}$ are identical with the "open circuit" (Carnot) values for the "active" differences $T_{1},-T_{2}$, and $\mu_{1},-\mu_{2}$, we may write

$$
\begin{aligned}
& T_{1^{\prime}}-T_{2^{\prime}}=T_{1}-T_{2}-I_{S}\left(R_{1 S}+R_{2 S}\right) \\
& \mu_{1^{\prime}}-\mu_{2^{\prime}}=\mu_{1}-\mu_{2}-I_{e}\left(R_{1 e}+R_{2 e}\right),
\end{aligned}
$$

where $I_{\mathrm{s}}$ and $I_{\mathrm{e}}$ are the conserved currents of entropy and electricity flowing through the energy-generating zone of the system. The indices 1 and 2 refer, respectively, to the resistances in the "upper" and "lower" part of the engine system.

Active (power producing) driving forces corresponding with Eqs. (10) and (11) are the temperature difference and electrochemical affinity $\mu_{1^{\prime}}-\mu_{2^{\prime}}$. Total power yield is

$$
\begin{aligned}
& p=\left(T_{1^{\prime}}-T_{2^{\prime}}\right) I_{\mathrm{s}}+\left(\mu_{1^{\prime}}-\mu_{2^{\prime}}\right) I_{\mathrm{e}}= \\
& \left(T_{1}-T_{2}\right) I_{\mathrm{s}}+\left(\mu_{1}-\mu_{2}\right) I_{\mathrm{e}}-\left(R_{1 \mathrm{~s}}+R_{2 \mathrm{~s}}\right) I_{s}^{2}-\left(R_{\mathrm{le}}+R_{2 \mathrm{e}}\right) I_{e}^{2}
\end{aligned}
$$

In terms of the definitions of total resistances Eq. (12) can be written in the form

$$
\begin{aligned}
& p=\left(T_{1^{\prime}}-T_{2^{\prime}}\right) I_{\mathrm{s}}+\left(\mu_{1^{\prime}}-\mu_{2^{\prime}}\right) I_{\mathrm{e}} \\
& =\left(T_{1}-T_{2}\right) I_{\mathrm{s}}+\left(\mu_{1}-\mu_{2}\right) I_{\mathrm{e}}-R_{s} I_{s}^{2}-R_{\mathrm{e}} I_{e}^{2}
\end{aligned}
$$

Equation (13) can easily be generalized to the case of coupled heat and charge transfer in the dissipative conductors of the system. Such a generalization will lead us to a general result for power limits in linear systems. The generalization has the form

$$
\begin{aligned}
& p=\left(T_{1^{\prime}}-T_{2^{\prime}}\right) I_{\mathrm{s}}+\left(\mu_{1^{\prime}}-\mu_{2^{\prime}}\right) I_{\mathrm{e}}= \\
& \left(T_{1}-T_{2}\right) I_{\mathrm{s}}+\left(\mu_{1}-\mu_{2}\right) I_{\mathrm{n}}-R_{\mathrm{ss}} I_{s}^{2}-R_{\mathrm{ss}} I_{s} I_{e}-R_{\mathrm{ee}} I_{e}^{2}
\end{aligned}
$$

After introducing the enlarged flux vector $\tilde{\mathbf{I}}=\left(I_{\mathrm{s}}, I_{\mathrm{e}}\right)$, the enlarged thermal potential vector $\tilde{\boldsymbol{\mu}}=(T, \mu)$ and the resistance tensor $\tilde{\mathbf{R}}$, Eq. (14) can be written in a simple and concise matrix-vector form

$$
p=\left(\tilde{\boldsymbol{\mu}}_{1}-\tilde{\boldsymbol{\mu}}_{2}\right) \cdot \tilde{\mathbf{I}}-\tilde{\mathbf{R}}: \tilde{\mathbf{I}} \tilde{\mathbf{I}}
$$

\section{Power Bounds for Chemical Engines and Fuel Cells}

We can now consider power bounds for chemical systems and fuel cells. Saving the paper's volume, we refer the reader to refs. [8-11] for performance bounds in chemical systems. While the dimensionality of potential vector $\tilde{\boldsymbol{\mu}}$ will certainly be much larger in real systems, the structure of Eq. (10) will be preserved for this equation written in the vector form. Maximum power corresponds with the vanishing partial derivatives

$$
\partial p / \partial \tilde{\mathbf{I}}=\tilde{\boldsymbol{\mu}}_{1}-\tilde{\boldsymbol{\mu}}_{2}-2 \tilde{\mathbf{R}} . \tilde{\mathbf{I}}=0
$$

Therefore, the optimal (power-maximizing) vector of currents at the maximum point of the system can be written in the form

$$
\tilde{\mathbf{I}}_{m p}=\frac{1}{2} \tilde{\mathbf{R}}^{-1} \cdot\left(\tilde{\boldsymbol{\mu}}_{1}-\tilde{\boldsymbol{\mu}}_{2}\right) \equiv \frac{1}{2} \tilde{\mathbf{I}}_{F}
$$

This result means that in the strictly linear systems the power-maximizing current vector $\tilde{\mathbf{I}}_{m p}$ is equal to one half of the purely dissipative current at the FourierOnsager point, $\tilde{\mathbf{I}}_{m p}$. The latter point refers to the system's state at which no power production occurs. Consistently, Eqs. (15) and (17) yield the following result for the maximum power limit of the system

$$
p_{m p}=\frac{1}{4}\left(\tilde{\boldsymbol{\mu}}_{1}-\tilde{\boldsymbol{\mu}}_{2}\right) \cdot \tilde{\mathbf{R}}^{-1} \cdot\left(\tilde{\boldsymbol{\mu}}_{1}-\tilde{\boldsymbol{\mu}}_{2}\right)
$$

In terms of the purely dissipative flux vector at the Fourier-Onsager point, $\tilde{\mathbf{I}}_{F}$, the above limit of maximum power is represented by an equation

$$
p_{m p}=\frac{1}{4} \tilde{\mathbf{R}}: \tilde{\mathbf{I}}_{F} \tilde{\mathbf{I}}_{F}
$$

On the other hand, power dissipated at the FourierOnsager point equals

$$
p_{F}=\tilde{\mathbf{R}}: \tilde{\mathbf{I}}_{F} \tilde{\mathbf{I}}_{F}
$$

Comparison of Eqs. (19) and (20) proves that, in linear thermo-electro-chemical systems, only at most $25 \%$ of power which is dissipated in the natural transfer process, can be transformed into the noble form of the mechanical power. This is a general result which, probably, cannot be easily generalized to the nonlinear transfer systems where significant deviations from Eq. (19) may appear 
depending on the nature of diverse nonlinearities. In fact, only forms of Eqs. (14) and (15) are sufficient to claim that the thermal force formula and the power formula for the thermal engine are similar to the voltage and power formulae in the fuel cell system. This proves a link between the mathematics thermal engines and fuel cells, thus the theory of fuel cells can be unified with the theory of thermal engines.

\section{Some Experimental Data for Fuel Cells at Steady States}

Voltage lowering in fuel cells below the reversible voltage is a good measure of their imperfection. Yet we need to distinguish between Nernst ideal voltage $E^{0}$ or and idle run voltage, $E_{0}$. It is the latter quantity from which all rate dependent losses of voltage should be subtracted. A number of approaches for calculating these polarization losses have been reviewed in literature by Zhao, Ou and Chen [12]. The details of calculations of the idle run voltage $E_{0}$ are discussed by Wierzbicki [13] who has implemented the Aspen Plus ${ }^{\mathrm{TM}}$ software to investigate the SOFC based energy systems using his own theoretical model of power yield kinetics. The model was based on Eqs. (22)-(25) of the present paper and some associated relationships. His calculations were compared with the experimental findings of the voltage and power in a laboratory FC system.

The basic structure of fuel cells includes electrolyte layer in contact with a porous anode and cathode on either side. Gaseous fuels are fed continuously to the anode (negative electrode) compartment and an oxidant (i.e., oxygen from air) is fed to the cathode (positive electrode) compartment. Electrochemical reactions at the electrodes produce an electric current. Voltage lowering in fuel cells below the reversible value is a good measure of their imperfection, Fig.4. With the concept of effective nonlinear resistances operating voltage of a fuel cell can be represented as the departure from the idle run voltage $E_{0}$

$$
V=E_{0}-V_{\text {int }}=E_{0}-V_{a c t}-V_{c o n c}-V_{\text {ohm }}
$$

The losses, which are called polarization, include three main sources: activation polarization $\left(V_{\text {act }}\right)$, ohmic polarization $\left(V_{\mathrm{ohm}}\right)$, and concentration polarization $\left(V_{\text {conc }}\right)$. Large number of approaches for calculating polarization losses has been reviewed [12]. As voltage losses increase, the increasing power finally drops for sufficiently large $i$, i.e power maxima appear (Fig. 4).

In some situations difference between $E^{0}$ and $E_{0}$ is a current independent loss which may be described by a fraction $\Xi$ characterizing the detrimental increase of chemical potentials of products caused by their dilution by un-reacted substrates. With the concept of effective nonlinear resistances operating voltage can be represented as the departure from the idle run voltage $E_{0}$ (the quantity which replaces the reversible voltage $E^{0}$ in more involved situations)

$$
\begin{aligned}
& V=E_{0}-V_{\mathrm{int}}=E_{0}-V_{a c t}-V_{c o n c}-V_{\text {ohm }} \\
& =E_{0}-i\left(R_{a c t}+R_{c o n c}+R_{\text {ohm }}\right)
\end{aligned}
$$

(Note the analogy between this equation and Eqs (13) and (14)). The losses, called polarization, include three main sources: activation polarization $\left(V_{\text {act }}\right)$, ohmic polarization $\left(V_{\mathrm{ohm}}\right)$, and concentration polarization $\left(V_{\text {conc }}\right)$. They refer to the equivalent activation resistance $\left(R_{\text {act }}\right)$, equivalent ohmic resistance $\left(R_{\mathrm{ohm}}\right)$, and equivalent concentration resistance $\left(R_{\text {conc }}\right)$. Activation and concentration polarization occur at both anode and cathode locations, while the resistive polarization represents ohmic losses throughout the fuel cell. Activation polarization $V_{\text {act }}$ is neglected in the model of ref. [13], nonetheless the power curve is typical. Since the voltage losses increase with current, the initially increasing power begins finally to decrease for sufficiently large currents, so that maxima of power are observed (Fig. 4). The data include the losses of the idle run attributed to the flaws in electrode constructions and other imperfections which cause that the open circuit voltage is in reality lower than $E^{0}$. Moreover, in the literature there are many other experimental and theoretical examples showing power maxima in fuel cells and proving the suitability of the thermal machine theory to chemical and electrochemical systems.

Voltage-power-current characteristics of SOFC

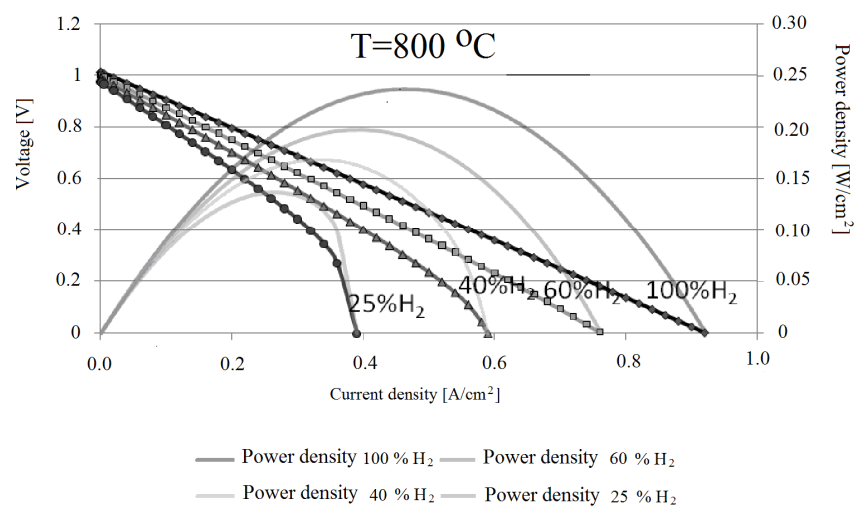

Fig.4. Voltage-current density and power-current density characteristics of the SOFC for various fuels at the temperature $800{ }^{\circ} \mathrm{C}$. Continuous lines represent the Aspen Plus ${ }^{\mathrm{TM}}$ calculations testing the model versus the experiments [13].

A voltage equation used in Wierzbicki's SOFC calculations is:

$$
V=E_{0}\left(T, p_{H_{2}}\right)-i A_{R}\left(p_{H_{2}}\right) \exp \left(\frac{\Delta E}{R T}\right)+B \ln \left(1-\frac{i}{i_{L}\left(T, p_{H_{2}}\right)}\right)
$$

where a limiting current is introduced defined by the equation

$$
i_{L}=C_{1} T^{-1} \exp \left(\frac{-E_{a}}{R T}\right) p_{H_{2}}
$$


in which $C_{1}$ is a experimentally determined parameter. Power density is simply the product of voltage $V$ and current density $i$

$$
p=V i
$$

In an ideal situation (no losses) the cell voltage is defined by the Nernst equation. Yet, while the first term of Eq. (23) defines the voltage without load, it nonetheless takes into account the losses of the idle run, which are the effect of flaws in electrode constructions and other imperfections which cause that the open circuit voltage in reality to be lower than the theoretical value. The losses include ohmic polarization and concentration polarization. The second term of Eq. (23) quantifies ohmic losses associated with electric resistance of electrodes and flow resistance of ions through the electrolyte. The third term refers to mass transport losses. Quantity $i_{\mathrm{L}}$ is the particular current density arising when the fuel is consumed in the reaction with the maximum possible feed rate.

\section{Conclusion}

The FC model developed in this paper describes physical and chemical performance of the irreversible fuel cells at various operating conditions. Lowering of SOFC efficiency is linked with polarizations (activation, concentration and ohmic) and incomplete conversions. Effect of incomplete conversion has been modeled assuming that substrates can be remained after the reaction and that side reactions may occur. Optimum and feasibility conditions have been obtained for a fuel cell, and discussed for some input parameters such as efficiency, power output, and electric current density of the cell. Calculations of optimal power show that the data differ for power generated and consumed, and depend on parameters of the system, e.g., current intensity, number of mass transfer units, polarizations, electrode surface area, average chemical rate, etc. These data provide bounds for SOFC generators that are more exact than reversible bounds for electrochemical transformation.

Power production bounds (limits) obtained in this paper are enhanced in comparison with those predicted by classical thermodynamics. As opposed to classical thermodynamics, our power bounds depend not only on changes of the thermodynamic state of participating resources but also on process irreversibilities, process direction and mechanism of heat and mass transfer. In fact, our research provides enhanced bounds. In thermostatics the bound on the work produced coincides with that on the work consumed. The generalized thermo-kinetic bounds, obtained here, are stronger than those predicted by thermostatics. Only for infinitely long durations or for processes with excellent transfer (an infinite number of transfer units) the thermokinetic bounds reduce to the classical thermostatic bounds [14].

Because of the presence of both static and kinetic terms in converter modeling, this research provides data for power production limits which are enhanced in comparison with those found from the classical thermodynamics. Classical thermostatic bounds are often too far from reality to be really useful. Generalized bounds are stronger than classical; they depend not only on state changes but also on process irreversibilities, ratios of stream flows, stream directions, and mechanism of heat and mass transfer. The methodology familiar for thermal machines has been extended here to chemical and electrochemical engines. Extensions are also available for multicomponent, multireaction units [11]. Thus, with non-equilibrium thermodynamics, we can confront and surmount the limitations of applying classical thermodynamics to real processes. This is a direction with many opportunities, especially for separation and chemical systems.

\section{Acknowledgement}

This research was supported in part by Polish Ministry of Science, grant NN208 019434: Thermodynamics and Optimization of Chemical and Electrochemical Energy Generators with Applications to Fuel Cells. Results in Fig. 4 were found with M. Wierzbicki during the present author's supervising of his MsD thesis. Thesis cosupervising by $\mathrm{dr}$ J. Jewulski of Warsaw Institute of Energetics is also appreciated.

\section{References}

[1] Sieniutycz, S., 2010. "Power Generation Limits in Thermal, Chemical and Electrochemical Systems", International conference on renewable energy and power quality (ICREPQ'10), Granada (Spain), 23th to 25th March, 2010, paper on http://www.icrepq.com.

[2] Curzon, F.L., Ahlborn, B., 1975. "Efficiency of Carnot engine at maximum power output". American J. Phys., 43(1), 22-24.

[3] De Vos, A., 1994. Endoreversible Thermodynamics of Solar Energy Conversion, Oxford University Press, pp. 30-41.

[4] Sieniutycz, S., Kuran, P., 2005. "Nonlinear models for mechanical energy production in imperfect generators driven by thermal or solar energy", Intern. J. Heat Mass Transfer, 48(3-4), 719-730.

[5] Sieniutycz, S., Kuran, P., 2006. "Modeling thermal behavior and work flux in finite-rate systems with radiation". Intern. J. Heat and Mass Transfer, 49(17-18), 3264-3283.

[6] Sieniutycz, S., 2009. "Dynamic programming and Lagrange multipliers for active relaxation of resources in nonequilibrium systems. Applied Mathematical Modeling, 33(3), 1457-1478.

[7] Kuran, P., 2006. Nonlinear Models of Production of Mechanical Energy in Non-Ideal Generators Driven by Thermal or Solar Energy, Thesis (PhD). Warsaw University of Technology.

[8] Sieniutycz, S., Jeżowski, J., 2009. Energy Optimization in Process Systems. Oxford: Elsevier, pp.331-358.

[9] Sieniutycz, S., 2008. "An analysis of power and entropy generation in a chemical engine". Intern. J. of Heat and Mass Transfer, 51(25-26), 5859-5871.

[10] Tsirlin, A. M., Kazakov, V., Mironova, V. A., Amelkin, S. A., 1998. "Finite-time thermodynamics: conditions of minimal dissipation for thermodynamic process". Physical Review E, 58 (1), 215-223. 
[11] Sieniutycz, S., 2009. "Complex chemical systems with power production driven by mass transfer". Intern. J. of Heat and Mass Transfer, 52(10), 2453-2465.

[12] Zhao, Y., Ou, C., Chen, J., 2008. "A new analytical approach to model and evaluate the performance of a class of irreversible fuel cells". International Journal of Hydrogen Energy, 2008 (33), 4161- 4170.

[13] Wierzbicki, M., 2009. Optimization of SOFC based energy system using Aspen Plus ${ }^{T M}$, Thesis (MsD) supervised by S. Sieniutycz (Faculty of Chemical and Process Engineering, Warsaw University of Technology) and J. Jewulski (Laboratory of Fuel Cells, Institute of Energetics, Warsaw.

[14] Berry, R. S., Kazakov, V. A., Sieniutycz, S., Szwast, Z., Tsirlin, A. M., 2000. Thermodynamic Optimization of Finite Time Processes, Chichester, Wiley, p.197. 